\title{
The multi-object optimization of a medicine delivery system of Ebola
}

\author{
Yue Song ${ }^{1, a}$ \\ ${ }^{1}$ School of North China Electric Power University, Baoding 071000, China. \\ a15733229653@163.com
}

Keywords: the Epidemic Model SIQR, The Analytic Hierarchy Process, Ebola

\begin{abstract}
After analyzing the data from WHO, we choose Guinea to be an example to simulate our model. We first predict the trend of infected people by using the Epidemic Model SIQR. After analyzing the epidemic of Guinea, we learn that Ebola in Guinea has reached its maximum and it is in decline now. So we determine the demand of medicine according to the current situation. Then, we summarize the data and use the Analytic Hierarchy Process to find out 3 distribution centers from 6 heavily infected cities with respect to four criterion which are the most critical. In the final part of the basic model, we take a numerical example to validate the availability of our model and the agreement between the mathematical experiment and the reality shows the rationality of our model.
\end{abstract}

\section{Introduction}

Ebola virus which is a deadly disease discovered in 1976, the first outbreak has killed kills 50 to 90 percent of the infected people. nearly 300 people. Unfortunately, the virus broke out again in 2014 and has caused much more deaths. During the time without drugs, people with Ebola were treated with only general therapies. They might be given much water or some treatments aimed at maintaining blood pressure and oxygen levels. In this case, the United Nations World Health Organization (WHO) has attached great importance to it. To establish a delivery system which makes optimal allocation of the medicine and cost least is a pressing job. Making efficient distribution to the most infected area is our purpose in this paper. From data in the website of WHO, Ebola are most prevalent in Guinea, Liberia and Sierra Leone (details in Figure 1). We take Guinea as an example to capture our delivery system. Assume that the intellectual property has been protected well, the drug production can be mastered or specialists are sent to places where the medicine is needed. (To make sure factories can be built at needed places and are authorized to manufacture this medicine.) We approach our delivery system by first building differential equations according to the Epidemic Model SIQR and get the curve of infected people. We validate that the curve conforms with the scatter distribution well and draw a conclusion that the infected people have no trend to grow up during a short term. So we can determine the demand of medicine according to the current number of infected people without worrying about serious shortage of medicine supply caused by another break-out. Next, we use the Analytic Hierarchy Process to find out three distribution centers from six infected cities of Guinea. After that, we use linear programming to ensure that the needs are met and get the minimum cost when the medicine is in short supply. In the end, we consider the influence of the transportation time and transportation cost and optimize our model by using multi-objective programming to determine the means of transportation.

\section{The Epidemic Diffusion Model}

This model bases on the Epidemic Model SIQR.[2]

Since the high rate of death caused by Ebola, the number of death should be taken account. residents were divided into 4 types: healthy people (S), infected people (I), quarantined people (Q) and removed people who were recovered or dead because of Ebola $(\mathrm{R})$. Then, respectively define the percentage of each para -meter relative to the total population $\mathrm{N}$ as $\mathrm{s}(\mathrm{t}), \mathrm{i}(\mathrm{t})$, $\mathrm{q}(\mathrm{t})$ and $\mathrm{r}(\mathrm{t})$. So, 


$$
s(t)+i(t)+q(t)+r(t)=1
$$

Other parameters defined:

$\alpha$ : the average number of people which the patients contact with per day;

$\beta$ : the percentage of people who were cured or dead because of Ebola relate to the infected people; $\gamma$ : the percentage of quarantined people relate to the infected people.

$$
\left\{\begin{array}{l}
\frac{d q}{d t}=\gamma i \\
\frac{d r}{d t}=\beta i \\
\frac{d i}{d t}=\alpha s i-\beta i-\gamma i, i(0)=i_{0} \\
\frac{d s}{d t}=-\alpha s i, s(0)=s_{0}
\end{array}\right.
$$

With equations (1), we capture :

$$
\frac{d i}{d s}=\frac{\beta+\gamma}{\alpha s}-1,\left.i\right|_{s=s_{0}}=i_{0}
$$

And then, we can get the result from formula (2):

$$
i=\left(s_{0}+i_{0}\right)-s+\frac{\beta+\gamma}{\alpha} \ln \frac{s}{s_{0}}
$$

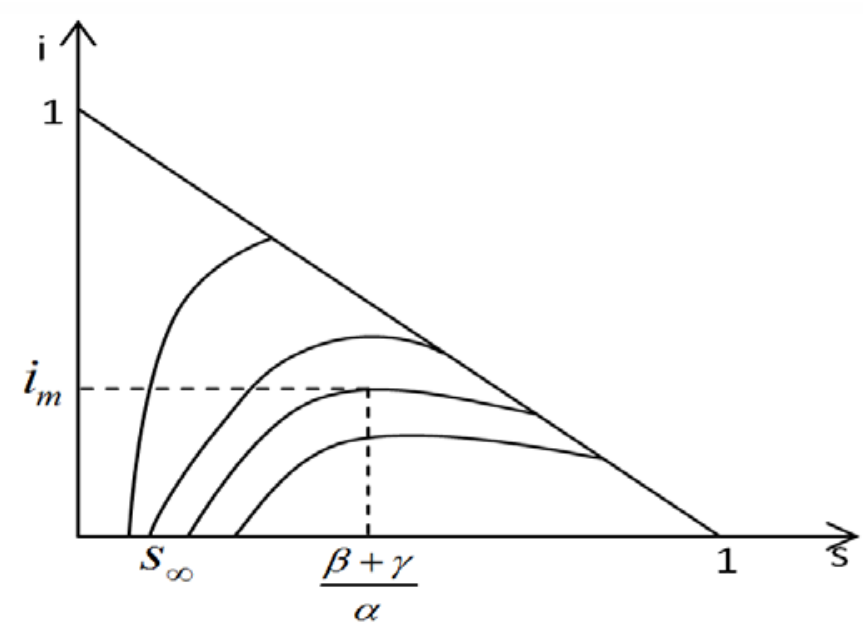

Figure 1: The phase orbit of the rate of healthy people and the rate of infective people

As Figure 1 shows, the curve made by Formula (4) is the phase orbit. We get the following conclusions from it:

1.The percentage of healthy people tends to be $s_{\infty}$ in the end. On the basis of the equation $\mathrm{i}=0$, we learn that $s_{\infty}$ is the root of the equation: (4). That is to say, $s_{\infty}$ is the abscissa of the point of intersection of the phase orbit curve and the horizontal axis.

2. Make the calculation: $\frac{\beta+\gamma}{\alpha s}-1=0$, and we get that $s=\frac{\beta+\gamma}{\alpha}$.

3. If $s_{o}>\frac{\beta+\gamma}{\alpha}, \mathrm{i}(\mathrm{t})$ will increase first and meet its maximum value at $s=\frac{\beta+\gamma}{\alpha}$. Namely, 


$$
i_{m}=s_{o}+i_{o}-\frac{\beta+\gamma}{\alpha}\left(1+\ln \frac{\alpha}{\beta+\gamma} s_{o}\right)
$$

After that,i(t) will begin to drop and have a tendency to 0 while $\mathrm{s}(\mathrm{t})$ will decrease monotonically to $S_{\infty}$.

Compared with the current situation of Guinea shown in Figure 2, we can draw a conclusion that Ebola virus has reached its maximum in Guinea and it is in decline now. That is to say, infected people won't increase in the near future. So we can determine the demand of medicine according to the current number of infected people without worrying a shortage of medicine supply caused by another break-out.

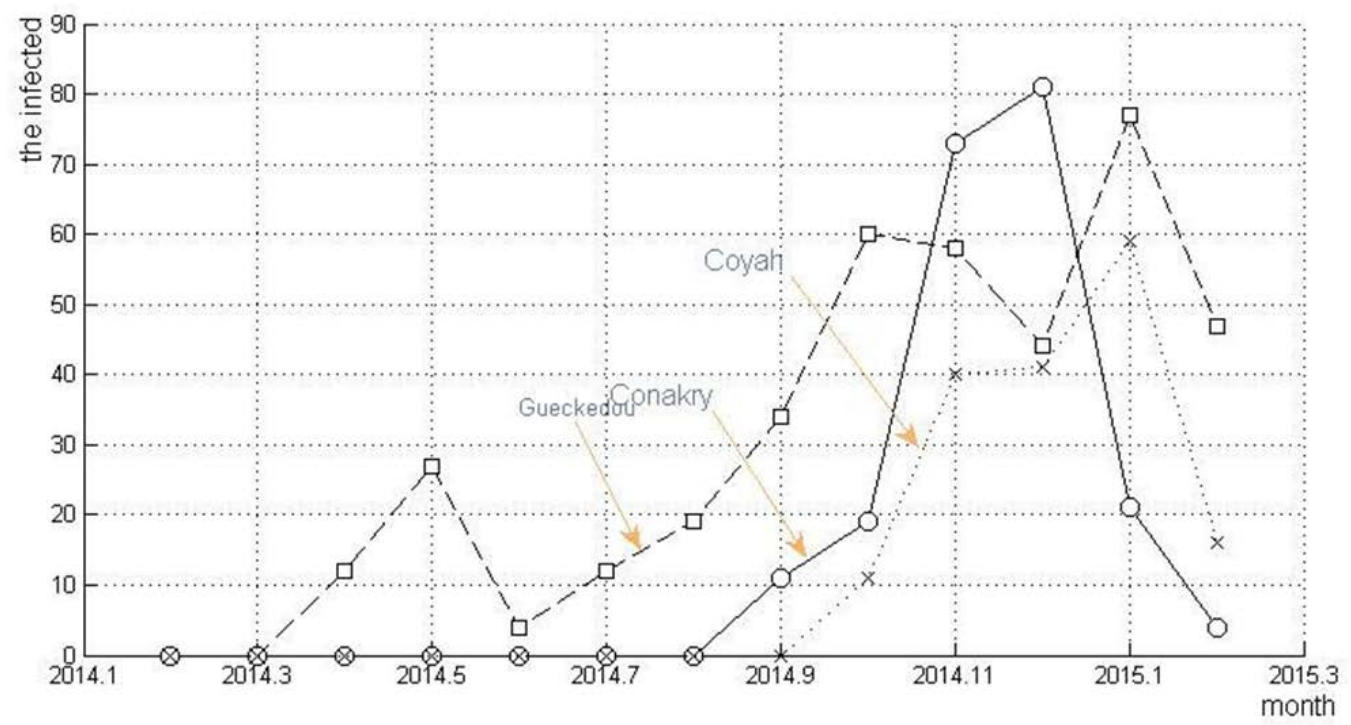

Figure 2: The current situation about Ebola in Guinea

\section{Transportation Program}

\subsection{Infected city chosen}

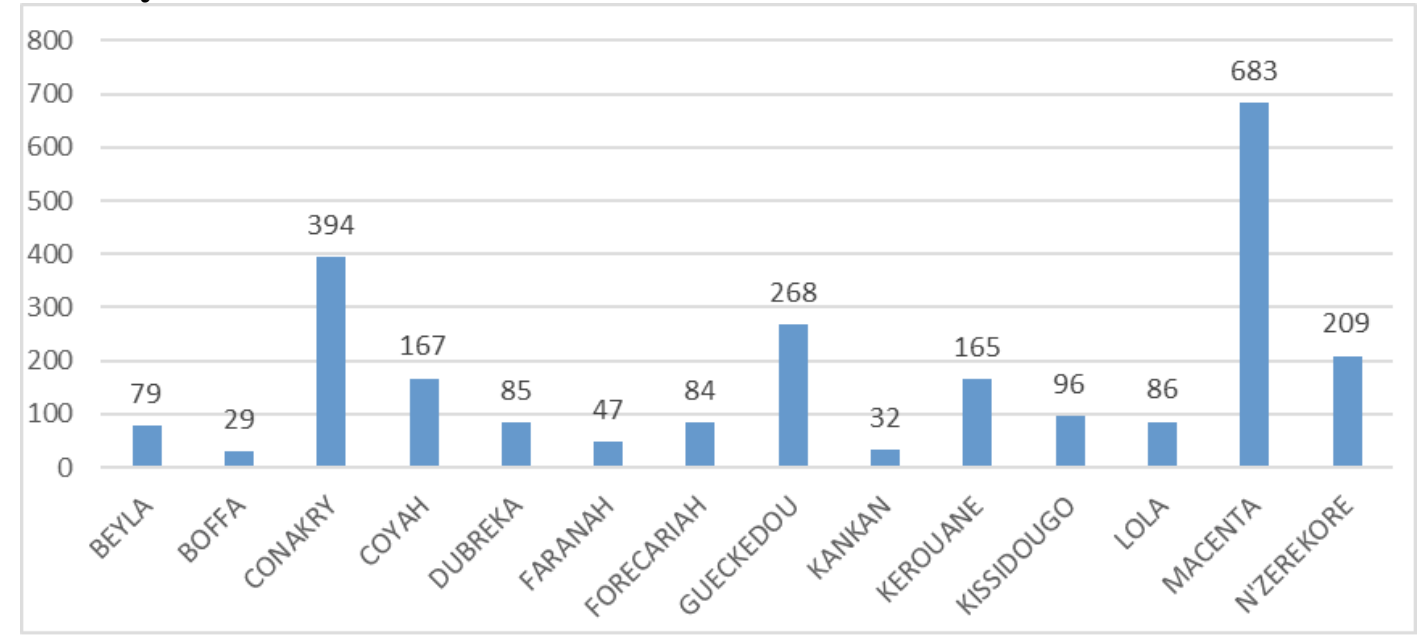

Figure 3: Number of the infected people in different cities of Guinea, data from WHO: http://apps.who.int/ebola/en/ebola-situation-report/situationreports/ebola-situation-report-4-february $-2015$

From Figure 3, we learn that infected people in Guinea mainly distributed in 6 cities: Macenta, Conakry, Gueckedou, N’Zerekore, Coyah and Kerouane. 


\subsection{Distribution centers chosen by using the Analytic Hierarchy}

We select these 6 cities to be the infected areas where need the medicine and use the Analytic Hierarchy Process to find out 3 of them to be the distribution centers.

Based on the calculation result, Macenta, with a priority of 0.2739 ,is the most suitable distribution center. Conakry, with a priority of 0.2256,is second, and Gueckedou, is third. Thus, the three distribution centers are:Macenta, Conakry, Gueckedou.

\section{Transportation Program}

The following results show the validation and the accuracy of our model:

- It costs much more to deliver medicine to Conakry and Coyah than to other cities. In fact, these two cities locate in a relatively remote location.So it is reasonable.

- The distributions themselves get the largest amount of medicine. It is not difficult to know that distribution centers should first satisfy the demand of itself before delivering out when the medicine is in short supply.

- Since Conakry is the capital of Guinea, both the supply and the demand of Conakry have great influence on the objective function.

And we can also get the conclusion that the number of medicine which deliver from Macenta to Conakry has the greatest impact on the objective function.

\section{Summary}

We predict the trend of Ebola virus by the SIQR model and get the demand of medicine. Then, we discover that the number of medicine which delivered from Macenta to Conakry has the greatest impact on the cost. Since Conakry is the capital of Guinea, both the supply and the demand of Conakry have great inuence on the cost. So when it is possible to increase production capacity, the increasing production of Conakry must be considered first. Of course, each distribution center should first satisfy the demand of itself when the medicine is in short supply. From the last model,we conclude that we should try to use air transport when the city has enough airports and avoid transporting to distant cities.

\section{References}

[1]. http://www.who.int/en/ [2015-2-9]

[2]. Qiyuan Jiang, Jinxing Xie, Yun Ye. Mathmetical Model. Higher Educational Press. China Higher Education Press, 2008, p.135-143.

[3]. http://en.wikipedia.org/wikiTransport in Guinea\#Railways

[4]. http://www.google.cn/maps/@8.6376408,-9.7432236,9z

[5]. Xianghe Jing, Dongfeng Wei, Xianzhong Zhou: Hybrid genetic algorithm for multi-object optimization of transportation modes in multimode transportation(Computer Engineering and Applications, China 2008),p.210-212. 